\title{
ON THE ANALYTICAL SOLUTION OF A TWO-PLACE FUNCTIONAL EQUATION
}

\author{
El-Sayed El-Hady \\ Basic Sciences Department, Faculty of Computers and Informatics, Suez Canal University, Ismailia, \\ Egypt \\ Elsayed_Elhady@ci.suez.edu.eg
}

Received $23 / 2 / 2017$

Revised 4/4/2017

Accepted 6/9/2017

\begin{abstract}
The main objective of this paper is to investigate the analytical solution of a special case of the general class of challenged functional equations given by

$$
A_{1}(x, y) f(x, y)=A_{2}(x, y) f(x, 0)+A_{3}(x, y) f(0, y)+A_{4}(x, y) f(0,0)+A_{5}(x, y),
$$

where $A_{i}(x, y), i=1, \ldots, 5$ are given functions in two complex variables $x, y$. The main unknown function $f(x, y)$ is defined in such a way that for every fixed $x$ it is analytic in the $y$-unit disk, and similarly for $y$. The unknown function $f(x, 0)$ is defined as $f(x, 0): D \rightarrow \mathbb{C}$, where $D$ stands for the unit disk, and a similar definition holds for the other unknown function $f(0, y)$. The functional equation considered in this article is not solved yet. However it is an interesting equation as it arose from a queueing model of a network gateway linking two ethernet local area networks. The solution is obtained by reduction to Riemann-Hilbert boundary value problem via using conformal mappings.
\end{abstract}

keyword Functional equation, Two-variable, Queueing model, Complex analysis

Classification No: 30D05, 39B72, 65Q20, 93C30, 97I80

\section{Introduction}

Functional equations are a relatively old subject of mathematics see e.g. [1,2]. However, they have various recent applications in many fileds like e.g. in information theory see [3], in applied sciences see [4], in databases see [5], and in communications see $[6,7]$. This article is mainly concerned with a solution of a two-place functional equation arising from a network gateway queueing model originally published in [8]. The current functional equation is a special case of the general structure of functional equations introduced recently in [9]. It should be noted that in [10] the authors introduced a closed from solution of the equation of interest by utilizing to a great extent the knowledge of the physical properties of the underlying gateway. In the current article we introduce an analytic solution using only mathematical techniques. The solution of the equation of interest is obtained by using the theory of boundary value problems. It is worth stating that Malyshev [11] pioneered the approach of transforming functional equations to boundary value problems in the early 1970s. The idea to reduce functional equations for the generating function to a standard Riemann-Hilbert boundary value problem stems from the work of Fayolle and Iasnogorodski [12] on two parallel $M / M / 1$ queues with coupled processors.

Lion's share of boundary value problems for analytic functions exists in the book of Cohen and Boxma [13] and that of Fayolle et al. [14]. As far as we know the functional equation in [8] is not yet solved, and hence in the current article we investigate the solution using boundary value problems approach. We will solve the current functional equation by reduction to a boundary value problem of the following form: Find a function $h($.$) such that:$ 
1. $h($.$) is analytic in D^{+}$

2. $h($.$) is continuous in the closure \operatorname{cl}\left(D^{+}\right)$of the unit disk

3. $h($.$) satisfies$

$$
\Re(\mathrm{i} a(u) h(u))=b(u), u \in D,
$$

for some given hölder continuous functions $a($.$) and b($.$) with a \neq 0$ on $D$.

Since the function $h(u)$ is real on the real axis. This can be easily seen from the definition since, the unknown $h(u)$ is in general defined as

$$
h(u)=\sum_{k=0}^{\infty} p_{k} u^{k}, u \in \mathbb{C},
$$

for some real nonnegative coefficient $p_{k}$. Then it is possible to define by using the Schwartz reflection principle (see e.g. [15-18]), the function $g(u)=\overline{h(1 / \bar{u})}$, which is analytic in $D^{-}$. The function $h(u)($ resp. $g(u))$ is the restriction to $D$ (resp. $D^{-}$) of the sectionally analytic function (see e.g. [19]) $H(u$ ), which satisfies the following Riemann-Hilbert problem: Find a sectionally analytic function $H(u)$ with respect to the unit circle, bounded at infinity $(H(\infty)=h(0))$ such that for $u \in D$

$$
a(u) H^{+}(u)-a(u) H^{-}(u)=-2 i b(u),
$$

where $H^{+}(u)$ (resp. $H^{-}(u)$ ) is the interior (resp. exterior) limit of the function $H(u)$ on the unit circle. The solution of this problem, when it exists, is given by (see e.g. [20])

$$
H(u)=\frac{\phi^{+}(u)}{2 \pi i} \int_{D} \frac{B(z)}{\phi^{+}(z)(z-u)} d z+Q(u) \phi(u)
$$

where $Q(u)$ is some polynomial, which can be determined by using the conditions at infinity, the function $B(u)$ is given by

$$
B(u)=-2 \mathrm{i} \frac{b(u)}{a(u)}
$$

and the function $\phi(u)$ is defined by

$$
\phi(u)= \begin{cases}\exp \left(\frac{1}{2 i \pi} \int_{D} \log \left(z^{-\kappa} \frac{\overline{a(z)}}{a(z)}\right) \frac{d z}{z-u}\right) & \text { if } u \in D^{+} \\ \frac{1}{u^{\kappa}} \exp \left(\frac{1}{2 i \pi} \int_{D} \log \left(z^{-\kappa} \frac{\overline{a(z)}}{a(z)}\right) \frac{d z}{z-u}\right) & \text { if } u \in D^{-}\end{cases}
$$

with $\kappa$ denoting the index of the Riemann-Hilbert problem (the index of $\overline{a(z)} / a(z))$ and $\phi^{(+)}(u)$ being the interior limit of the function $\phi(u)$ on the unit circle.

The article is organized as follows: In section 2 we recall the functional equation from the original article [8], in section 3 we introduce some preliminary results including the analysis of the kernel, in section 4 an analytic continuation of the function defined by the kernel is proved, in section 5 we recall the idea of the resultant of two polynomials to compute the potential singularities of the two unknowns as done in a recent paper. The singularities play a crucial role in the construction of the boundary value problems, in section 6 we solve the functional equation by reduction to a special case of the boundary value problem stated in the introduction. In section 7 we conclude this article.

\section{The functional equation}

The article [8] ends up with the following two-place functional equation

$$
\begin{aligned}
& (M(x, y)-x y) f(x, y)=(1-y)\left(M(x, 0)+\tilde{r}_{1} \xi_{2} x y\right) f(x, 0) \\
& +(1-x)\left(M(0, y)+\tilde{r}_{2} \xi_{1} x y\right) f(0, y)-(1-x)(1-y) M(0,0) f(0,0),
\end{aligned}
$$

where

$$
M(x, y)=\left(\tilde{r}_{1}+r_{1} \tilde{s}_{1} y+\xi_{1} x y\right)\left(\tilde{r}_{2}+r_{2} \tilde{s}_{2} x+\xi_{2} x y\right)
$$


and $0<r_{j}, s_{j}, \xi_{j}:=r_{j} s_{j}<1, j=1,2$. By using the above equation, we can write equation (3) in the form

$$
\begin{gathered}
\left(\left(\tilde{r}_{1}+r_{1} \tilde{s}_{1} y+\xi_{1} x y\right)\left(\tilde{r}_{2}+r_{2} \tilde{s}_{2} x+\xi_{2} x y\right)-x y\right) f(x, y) \\
=(1-y) \tilde{r}_{1}\left(\tilde{r}_{2}+r_{2} \tilde{s}_{2} x+\xi_{2} x y\right) f(x, 0)+(1-x) \tilde{r}_{2}\left(\tilde{r}_{1}+r_{1} \tilde{s}_{1} y+\xi_{1} x y\right) f(0, y) \\
-(1-x)(1-y) \tilde{r}_{1} \tilde{r}_{2} f(0,0) .
\end{gathered}
$$

The main unknown function $f(x, y)$ is defined in [8] as a probability generating function (PGF) of the sequence $p_{m, n}$

$$
f(x, y)=\sum_{m, n=0}^{\infty} p_{m, n} x^{m} y^{n},|x| \leq 1,|y| \leq 1
$$

while the marginal PGFs $f(x, 0)$ and $f(0, y)$ are defined as

$$
f(x, 0)=\sum_{m=0}^{\infty} p_{m, 0} x^{m},|x| \leq 1,
$$

and

$$
f(0, y)=\sum_{n=0}^{\infty} p_{0, n} y^{n},|y| \leq 1 .
$$

The solution of equation (4) will be investigated in the next sections.

\section{Preliminary results}

We introduce some results towards the reduction to a boundary value problem

\subsection{The set-up}

For simplicity reasons equation (4) can be written as

$$
A_{1}(x, y) f(x, y)=A_{2}(x, y) f(x, 0)+A_{3}(x, y) f(0, y)+A_{4}(x, y) f(0,0)
$$

where

$$
\begin{aligned}
& A_{1}(x, y)=\left(\tilde{r}_{1}+r_{1} \tilde{s}_{1} y+\xi_{1} x y\right)\left(\tilde{r}_{2}+r_{2} \tilde{s}_{2} x+\xi_{2} x y\right)-x y, \\
& =\xi_{2} \xi_{1} x^{2} y^{2}+r_{2} \tilde{s}_{2} \xi_{1} x^{2} y+\xi_{2} r_{1} \tilde{s}_{1} x y^{2}+\left(\tilde{r}_{1} \xi_{2}+r_{1} r_{2} \tilde{s}_{1} \tilde{s}_{2}+\tilde{r}_{2} \xi_{1}-1\right) x y \\
& +\tilde{r}_{1} r_{2} \tilde{s}_{2} x+r_{1} \tilde{r}_{2} \tilde{s}_{1} y+\tilde{r}_{1} \tilde{r}_{2} \text {, } \\
& A_{2}(x, y)=\tilde{r}_{1}(1-y)\left(\tilde{r}_{2}+r_{2} \tilde{s}_{2} x+\xi_{2} x y\right) \text {, } \\
& =\tilde{r}_{1} \tilde{r}_{2}+\tilde{r}_{1} r_{2} \tilde{s}_{2} x-\tilde{r}_{1} \tilde{r}_{2} y+\left(\tilde{r}_{1} \xi_{2}-\tilde{r}_{1} r_{2} \tilde{s}_{2}\right) x y-\tilde{r}_{1} \xi_{2} x y^{2} \\
& A_{3}(x, y)=\tilde{r}_{2}(1-x)\left(\tilde{r}_{1}+r_{1} \tilde{s}_{1} y+\xi_{1} x y\right) \\
& =\tilde{r}_{1} \tilde{r}_{2}+r_{1} \tilde{r}_{2} \tilde{s}_{1} y-\tilde{r}_{1} \tilde{r}_{2} x+\left(\tilde{r}_{2} \xi_{1}-r_{1} \tilde{r}_{2} \tilde{s}_{1}\right) x y-\tilde{r}_{2} \xi_{1} x^{2} y,
\end{aligned}
$$

and

$$
\begin{aligned}
A_{4}(x, y) & =\tilde{r}_{1} \tilde{r}_{2}(x-1)(1-y) \\
& =\tilde{r}_{1} \tilde{r}_{2} x+\tilde{r}_{1} \tilde{r}_{2} y-\tilde{r}_{1} \tilde{r}_{2} x y-\tilde{r}_{1} \tilde{r}_{2} .
\end{aligned}
$$

Since by definition the main function $f(x, y)$ is an analytic function in the unit disk, then this means that if $A_{1}(x, y)=0$, then also

$$
A_{2}(x, y) f(x, 0)+A_{3}(x, y) f(0, y)+A_{4}(x, y) f(0,0)=0 .
$$


Since the unknown $f(0,0) \neq 0$ (necessary for the stability of the underlying system), then equation (6) is equivalent to

$$
A_{2}(x, y) \frac{f(x, 0)}{f(0,0)}+A_{3}(x, y) \frac{f(0, y)}{f(0,0)}+A_{4}(x, y)=0 .
$$

Introducing new functions

$$
F(x):=\frac{f(x, 0)}{f(0,0)}, G(y):=\frac{f(0, y)}{f(0,0)} .
$$

By using (8) in (7) we get the following functional equation

$$
A_{2}(x, y) F(x)+A_{3}(x, y) G(y)+A_{4}(x, y)=0
$$

on the set

$$
\left\{(x, y): A_{1}(x, y)=0\right\} .
$$

Now the solution of equation (4) is reduced to the solution of the equation (9) in only two unknowns namely $F(x)$ and $G(y)$. It is obvious that it suffices to find one unknown and plug it in the above equation to find the other unknown as done in [21]. The next and basic step in solving the functional equation is the analysis of the kernel defined by

$$
\left\{(x, y): A_{1}(x, y)=M(x, y)-x y=0\right\} .
$$

First note that the current functional equation is somehow different from the equations that appear in the literature (see e.g. [22], [23], [21]) in the following facts:

1. The functions $A_{2}(x, y), A_{3}(x, y)$, and $A_{4}(x, y)$ are not related to each other unlike the case in [22].

2. It is not possible to find the unknown $f(0,0)$ by a direct substitution in the main functional equation unlike the case in [21]. This is because there are no values for $x$ or $y$ for which both $A_{2}(x, y)=0$ and $A_{3}(x, y)=0$.

3. We have a completely symmetric equation i.e. the approach applied to find $F(x)$ will exactly be applied to find $G(y)$ this is due to the complete symmetry of the kernel $A_{1}(x, y)$.

4. We don't know the unknown $f(0,0)$ unlike the case in [22].

5 . We have many parameters namely $r_{1}, r_{2}, s_{1}, s_{2}, \xi_{1}$ and $\xi_{2}$ which may complicate the analysis.

6. The contours $L_{1}$ and $L_{2}$ defined below are not circles unlike the case in [12].

7. The function $F(x)$ may have singularities in the domain $L_{1}^{+}$.

8. There is no explicit stability condition of the system producing the functional equation unlike the case in [24].

9. We have a different random walk (in the interior of the random walk there are transitions to the east, north east, and north) producing the functional equation so we cannot use the compensation approach unlike the cases in [25-28].

\subsection{Kernel analysis}

A crucial role (see e.g. [29], [30], and [12]) in the solution of our equation is played by the kernel given by

$$
\left\{(x, y): A_{1}(x, y)=\left(\tilde{r}_{1}+r_{1} \tilde{s}_{1} y+\xi_{1} x y\right)\left(\tilde{r}_{2}+r_{2} \tilde{s}_{2} x+\xi_{2} x y\right)-x y=0\right\} .
$$

From (10) the kernel can be written as

$$
\begin{gathered}
A_{1}(x, y)=\xi_{1} \xi_{2} x^{2} y^{2}+r_{2} \tilde{s}_{2} \xi_{1} x^{2} y+r_{1} \tilde{s}_{1} \xi_{2} x y^{2}+\left(\tilde{r}_{1} \xi_{2}+r_{2} \tilde{s}_{2} r_{1} \tilde{s}_{1}+\tilde{r}_{2} \xi_{1}-1\right) x y \\
+\tilde{r}_{1} r_{2} \tilde{s}_{2} x+\tilde{r}_{2} r_{1} \tilde{s}_{1} y+\tilde{r}_{1} \tilde{r}_{2}=0 .
\end{gathered}
$$

Since equation (11) is a biquadratic equation it can be considered as a quadratic equation in $x$ as a function of $y$, or as a quadratic equation in $y$ as a function of $x$. So we have

$$
A_{1}\left(x, y_{+}(x)\right)=A_{1}\left(x, y_{-}(x)\right)=0,
$$

and also

$$
A_{1}\left(x_{+}(y), y\right)=A_{1}\left(x_{-}(y), y\right)=0,
$$

where $x_{ \pm}(y)$ are the solutions of (11) when considered as a quadratic equation in $x$. Similarly, $y_{ \pm}(x)$ are the solution of (11) when considered as a quadratic equation in $y$. Now we have to study the two cases separately as follows. 


\subsubsection{The kernel as a quadratic equation in $x$}

If we consider equation (11) as an equation in $x$ it can be written as follows

$$
\begin{aligned}
\left(\xi_{1} \xi_{2} y^{2}+r_{2} \tilde{s}_{2} \xi_{1} y\right) x^{2} & +\left(r_{1} \tilde{s}_{1} \xi_{2} y^{2}+\left(\tilde{r}_{1} \xi_{2}+r_{2} \tilde{s}_{2} r_{1} \tilde{s}_{1}+\tilde{r}_{2} \xi_{1}-1\right) y+\tilde{r}_{1} r_{2} \tilde{s}_{2}\right) x \\
& +\tilde{r}_{2} r_{1} \tilde{s}_{1} y+\tilde{r}_{1} \tilde{r}_{2}=0
\end{aligned}
$$

or in the form

$$
\alpha(y) x^{2}+\beta(y) x+\gamma(y)=0
$$

where

$$
\begin{gathered}
\alpha(y)=\xi_{1} \xi_{2} y^{2}+r_{2} \tilde{s}_{2} \xi_{1} y, \\
\beta(y)=r_{1} \tilde{s}_{1} \xi_{2} y^{2}+\left(\tilde{r}_{1} \xi_{2}+r_{2} \tilde{s}_{2} r_{1} \tilde{s}_{1}+\tilde{r}_{2} \xi_{1}-1\right) y+\tilde{r}_{1} r_{2} \tilde{s}_{2},
\end{gathered}
$$

and

$$
\gamma(y)=\tilde{r}_{2} r_{1} \tilde{s}_{1} y+\tilde{r}_{1} \tilde{r}_{2}
$$

Equation (12) has two solutions $x$, in the form

$$
x_{ \pm}(y)=\frac{-\beta(y) \pm \sqrt{D_{1}(y)}}{2 \alpha(y)}
$$

where

$$
D_{1}(y)=\beta^{2}(y)-4 \alpha(y) \gamma(y)
$$

It is easy to see that

$$
D_{1}(-1)>0, D_{1}(0)>0, D_{1}(1)>0 \text {, and } \lim _{y \rightarrow \infty} D_{1}(y)=\infty .
$$

Lemma 3.1. The functions $x_{ \pm}(y)$ given by (14) have four real branch points $y_{i}, i=1, \ldots, 4$ such that

$$
0<y_{1}<y_{2}<1<y_{3}<y_{4}<\infty .
$$

Proof. Branch points are the zeros of the discriminant, $D_{1}(y)$, because as $y$ traverses a small circuit around $y_{i}, i=1, \ldots 4$, $D_{1}(y)$ does not return to its original value. The function $D_{1}$ is given by

$$
\begin{aligned}
D_{1}(y) & =\beta^{2}(y)-4 \alpha(y) \gamma(y) \\
& =r_{1}^{2} \tilde{s}_{1}^{2} \xi_{2}^{2} y^{4}+\left[2 \tilde{r}_{1} r_{1} \tilde{s}_{1} \xi_{2}^{2}+2 r_{1}^{2} r_{2} \tilde{s}_{1}^{2} \tilde{s}_{2} \xi_{2}-2 r_{1} \tilde{s}_{1} \xi_{2}-2 r_{1} \tilde{r}_{2} \tilde{s}_{1} \xi_{1} \xi_{2}\right] y^{3} \\
& +\left[4 r_{1} \tilde{r}_{1} r_{2} \tilde{s}_{1} \tilde{s}_{2} \xi_{2}+\tilde{r}_{1}^{2} \xi_{2}^{2}+r_{1}^{2} r_{2}^{2} \tilde{s}_{1}^{2} \tilde{s}_{2}^{2}+\tilde{r}_{2}^{2} \xi_{1}^{2}+1+2 \tilde{r}_{2} \xi_{1}\right. \\
& \left.-2 \tilde{r}_{1} \xi_{2}+r_{1} r_{2} \tilde{r}_{2} \tilde{s}_{1} \tilde{s}_{2} \xi_{1}-2 r_{1} r_{2} \tilde{s}_{1} \tilde{s}_{2}-2 \tilde{r}_{1} \tilde{r}_{2} \xi_{1} \xi_{2}\right] y^{2} \\
& +\left[2 \tilde{r}_{1}^{2} r_{2} \tilde{s}_{2} \xi_{2}+2 r_{1} \tilde{r}_{1} r_{2}^{2} \tilde{s}_{1} \tilde{s}_{2}^{2}+\tilde{r}_{1} r_{2} \tilde{r}_{2} \tilde{s}_{2} \xi_{1}-2 \tilde{r}_{1} r_{2} \tilde{s}_{2}\right] y+\left(r_{2} \tilde{r}_{1} \tilde{s}_{2}\right)^{2} .
\end{aligned}
$$

In order to locate the zeros of $D_{1}$ it is sufficient to investigate the zeros of the function $\beta(y)$. This is because at the zeros $\left(y_{i}^{\beta}, i=1,2\right)$ of $\beta(y)$, the function $D_{1}$ will be

$$
D_{1}\left(y_{i}^{\beta}\right)=-4 \alpha\left(y_{i}^{\beta}\right) \gamma\left(y_{i}^{\beta}\right)
$$

Clearly, from (13) $\beta(y)=0$ has two solutions:

$$
y_{1}^{\beta}=\frac{1-\tilde{r}_{1} \xi_{2}-r_{2} \tilde{s}_{2} r_{1} \tilde{s}_{1}-\tilde{r}_{2} \xi_{1}+\sqrt{\left(\tilde{r}_{1} \xi_{2}+r_{2} \tilde{s}_{2} r_{1} \tilde{s}_{1}+\tilde{r}_{2} \xi_{1}-1\right)^{2}-4 r_{1} \tilde{s}_{1} \xi_{2} \tilde{r}_{1} r_{2} \tilde{s}_{2}}}{2 r_{1} \tilde{s}_{1} \xi_{2}}>1
$$

and

$$
0<y_{2}^{\beta}=\frac{1-\tilde{r}_{1} \xi_{2}-r_{2} \tilde{s}_{2} r_{1} \tilde{s}_{1}-\tilde{r}_{2} \xi_{1}-\sqrt{\left(\tilde{r}_{1} \xi_{2}+r_{2} \tilde{s}_{2} r_{1} \tilde{s}_{1}+\tilde{r}_{2} \xi_{1}-1\right)^{2}-4 r_{1} \tilde{s}_{1} \xi_{2} \tilde{r}_{1} r_{2} \tilde{s}_{2}}}{2 r_{1} \tilde{s}_{1} \xi_{2}}<1
$$


It should be noted first that since $y_{1}^{\beta}>1$, and the functions $\alpha(),. \gamma($.$) are positive there then the function D_{1}($.$) is negative.$ For the other root $y_{2}^{\beta}$ we have $0<y_{2}^{\beta}<1$, this holds only for the system parameters for which the system is stable i.e. it holds only on

$$
\left\{r_{i}, s_{i}, \xi_{i}: \xi_{1}<1-r_{2}, \xi_{2}<1-r_{1}, i=1,2\right\}
$$

and in this case also both the functions $\alpha($.$) and \gamma($.$) are positive so that the function D_{1}($.$) using (18) is negative. It follows$ from the properties of polynomials that there exist $y_{1}, y_{2}, y_{3}$ and $y_{4}$ such that

$$
0<y_{1} \leq y_{2}^{\beta} \leq y_{2}<1<y_{3} \leq y_{1}^{\beta}<y_{4}<\infty,
$$

and $D_{1}\left(y_{i}\right)=0, i=1,2,3,4$. Also, by the properties of polynomials, $D_{1}(y)>0$ in $\left(-\infty, y_{1}\right) \cup\left(y_{2}, y_{3}\right) \cup\left(y_{4}, \infty\right)$ and $D_{1}(y)<0$ in $\left(y_{1}, y_{2}\right) \cup\left(y_{3}, y_{4}\right)$.

It is interesting to find out that selecting any random values of the system parameters $r_{i}, s_{i}, \xi_{i}, i=1,2$ satisfying the stability condition (19) we get that the root $y_{2}^{\beta}$ lies in the interval $(0,1)$.

Lemma 3.2. For every $y \in\left[y_{1}, y_{2}\right]$ we have $y \in \mathbb{R}$, and the two roots $x_{+}(y)$ and $x_{-}(y)$ given by

$$
x_{+}(y)=\frac{-\beta(y)+\sqrt{D_{1}(y)}}{2 \alpha(y)}, x_{-}(y)=\frac{-\beta(y)-\sqrt{D_{1}(y)}}{2 \alpha(y)},
$$

are complex conjugate. Hence, the interval $\left(y_{1}, y_{2}\right)$ is mapped by $y \mapsto x_{ \pm}(y)$ onto a contour $L_{1}$. Any point on such a contour satisfies

$$
|x(y)|^{2}=\frac{\tilde{r_{2}} r_{1} \tilde{s_{1}} y+\tilde{r_{1}} \tilde{r_{2}}}{\xi_{1} \xi_{2} y^{2}+r_{2} \tilde{s_{2}} \xi_{1} y} .
$$

Proof. Follows directly from the fact that the discriminant $D_{1}(y)$ is zero for $y=y_{1}$ and $y_{2}$ and negative for $y \in\left(y_{1}, y_{2}\right)$, which is symmetric with respect to the real line, see figure 1 . For a point $x(y)$ on the contour $L_{1}$ we have from (12) and Vieta's formula that

$$
\begin{aligned}
x_{+}(y) x_{-}(y)=x(y) \bar{x}(y) & =|x(y)|^{2} \\
& =\frac{\gamma(y)}{\alpha(y)}=\frac{\tilde{r}_{2} r_{1} \tilde{s}_{1} y+\tilde{r}_{1} \tilde{r}_{2}}{\xi_{1} \xi_{2} y^{2}+r_{2} \tilde{s}_{2} \xi_{1} y} .
\end{aligned}
$$

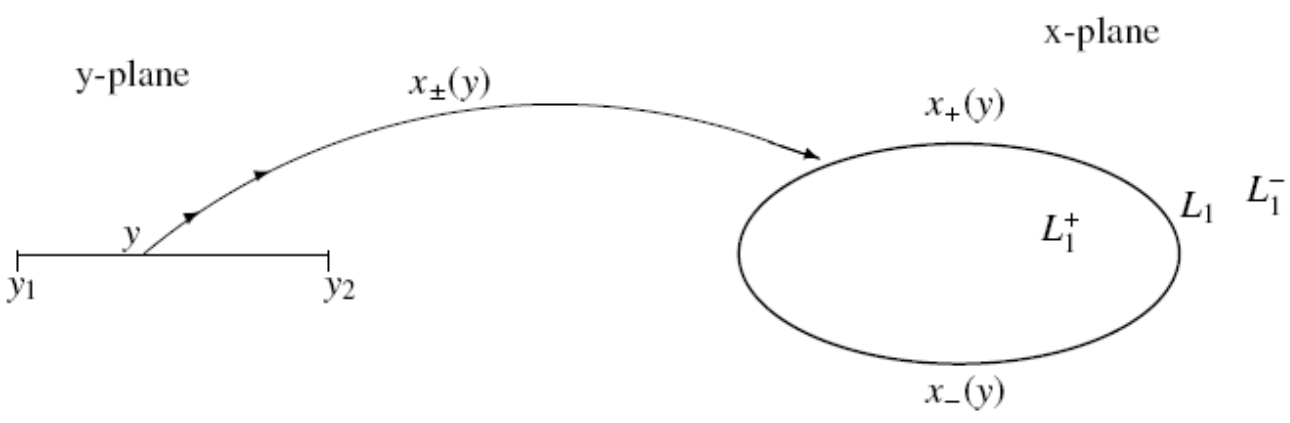

Figure 1: The function $x_{ \pm}(y)$ as a map from $\left[y_{1}, y_{2}\right]$ to the contour $L_{1}$ 
Remark 3.1. The functions $x_{ \pm}(y)$ defined by (14) have the following properties:

- They are local analytic functions except at zeros of the function $\alpha(y)$.

- They are well defined except at some algebraic branch points, which are the real zeros of the discriminant $D_{1}(y)$.

Remark 3.2. The function $x_{+}(y)$ has the following properties:

- It has removable singularities at $y=0$, and at $y=\frac{\xi_{2}-r_{2}}{\xi_{2}}$ which are the zeros of the function $\alpha(y)$, since it is easily verified that

$$
\lim _{y \rightarrow 0} x_{+}(y)=0, \quad \lim _{y \rightarrow \frac{\xi_{2}-r_{2}}{\xi_{2}}} x_{+}(y)=K
$$

for some finite value $K$.

- It can be expanded as a convergent power series of $y$ in some neighborhood of the points 0 , and at $y=\frac{\xi_{2}-r_{2}}{\xi_{2}}$, which both are removable singularities for the function $x_{+}(y)$.

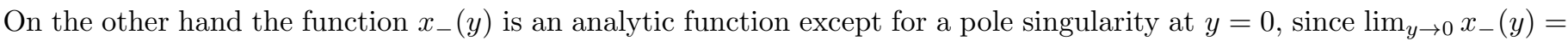
$-\infty$.

\subsubsection{The kernel as a quadratic equation in $y$}

If we consider equation (11) as a quadratic equation in $y$ it can be written as follows:

$$
\begin{gathered}
\left(\xi_{1} \xi_{2} x^{2}+r_{1} \tilde{s}_{1} \xi_{2} x\right) y^{2}+\left(r_{2} \tilde{s}_{2} \xi_{1} x^{2}+\left(\tilde{r}_{1} \xi_{2}\right.\right. \\
\left.\left.+r_{2} \tilde{s}_{2} r_{1} \tilde{s}_{1}+\tilde{r}_{2} \xi_{1}-1\right) x+\tilde{r}_{2} r_{1} \tilde{s}_{1}\right) y \\
+\tilde{r}_{1} r_{2} \tilde{s}_{2} x+\tilde{r}_{1} \tilde{r}_{2}=0
\end{gathered}
$$

or in the form

$$
\mu(x) y^{2}+\nu(x) y+\delta(x)=0,
$$

where

$$
\begin{gathered}
\mu(x)=\xi_{1} \xi_{2} x^{2}+r_{1} \tilde{s}_{1} \xi_{2} x, \\
\nu(x)=r_{2} \tilde{s}_{2} \xi_{1} x^{2}+\left(\tilde{r}_{1} \xi_{2}+r_{2} \tilde{s}_{2} r_{1} \tilde{s}_{1}+\tilde{r}_{2} \xi_{1}-1\right) x+\tilde{r}_{2} r_{1} \tilde{s}_{1},
\end{gathered}
$$

and

$$
\delta(x)=\tilde{r}_{1} r_{2} \tilde{s}_{2} x+\tilde{r}_{1} \tilde{r}_{2} .
$$

Equation (21) has two solutions in $y$, in the form

$$
y_{ \pm}(x)=\frac{-\nu(x) \pm \sqrt{D_{2}(x)}}{2 \mu(x)}
$$

where

$$
\begin{aligned}
D_{2}(x) & =\nu^{2}(x)-4 \mu(x) \delta(x) \\
& =r_{2}^{2} \tilde{s}_{2}^{2} \xi_{1}^{2} x^{4}+\left[2 r_{2} \tilde{r}_{2} \tilde{s}_{2} \xi_{1}^{2}+2 r_{1} r_{2}^{2} \tilde{s}_{2}^{2} \tilde{s}_{1} \xi_{1}-2 r_{2} \tilde{s}_{2} \xi_{1}-2 \tilde{r}_{1} r_{2} \tilde{s}_{2} \xi_{1} \xi_{2}\right] x^{3} \\
& +\left[4 r_{1} r_{2} \tilde{r}_{2} \tilde{s}_{1} \tilde{s}_{2} \xi_{1}+\tilde{r}_{2}^{2} \xi_{1}^{2}+r_{1}^{2} r_{2}^{2} \tilde{s}_{1}^{2} \tilde{s}_{2}^{2}+\tilde{r}_{1}^{2} \xi_{2}^{2}+1\right. \\
& \left.-2 \tilde{r}_{1} \xi_{2}-2 \tilde{r}_{2} \xi_{1}+\tilde{r}_{1} r_{1} r_{2} \tilde{s}_{2} \tilde{s}_{1} \xi_{2}-2 r_{2} r_{1} \tilde{s}_{2} \tilde{s}_{1}-2 \tilde{r}_{1} \tilde{r}_{2} \xi_{1} \xi_{2}\right] x^{2} \\
& +\left[2 r_{1} \tilde{r}_{2}^{2} \tilde{s}_{1} \xi_{1}+2 r_{1}^{2} r_{2} \tilde{r}_{2} \tilde{s}_{1}^{2} \tilde{s}_{2}+\tilde{r}_{2} r_{1} \tilde{r}_{1} \tilde{s}_{1} \xi_{2}-2 r_{1} \tilde{r}_{2} \tilde{s}_{1}\right] x \\
& +\left(r_{1} \tilde{r}_{2} \tilde{s}_{1}\right)^{2} .
\end{aligned}
$$

Lemma 3.3. The functions $y_{ \pm}(x)$ defined by $C_{1}(x, y)=0$, have four real branch points

$$
0<x_{1}<x_{2}<1<x_{3}<x_{4}<\infty
$$

Proof. Similar argument as in lemma 3.1 
Lemma 3.4. For each $x \in\left[x_{1}, x_{2}\right]$, the two roots $y_{+}(x)$ and $y_{-}(x)$ are complex conjugate. Hence, the interval $\left(x_{1}, x_{2}\right)$ is mapped by $x \mapsto y_{ \pm}(x)$ onto a closed contour $L_{2}$, which is symmetric with respect to the real line, fulfills

$$
|y(x)|^{2}=\frac{\tilde{r}_{1} r_{2} \tilde{s}_{2} x+\tilde{r}_{1} \tilde{r}_{2}}{\xi_{1} \xi_{2} x^{2}+r_{1} \tilde{s}_{1} \xi_{2} x} .
$$

Proof. Similar argument as in lemma 3.2

The functions $y_{ \pm}(x)$ defined by (22) have similar properties like in remarks 3.1 and 3.2.

\section{Analytic continuation}

Using the results of the previous section we assume that for $z \in \mathbb{C}, \arg (z) \in(-\pi, \pi]$, and we define the square root such that $\sqrt{x^{2}}=x$ if $x \geq 0$, and $\sqrt{-1}=i$. The couple $\left(y_{+}(x),\left(-\infty, x_{1}\right)\right)$ defines a germ of analytic function see [ [31], p. 42]. The following lemma shows how this germ can be analytically continued in the complex plane deprived of the segments $\left[x_{1}, x_{2}\right]$ and $\left[x_{3}, x_{4}\right]$. Let $z_{++}=\Re(z)+i|\Im(z)|$.

Lemma 4.1. The function

$$
Y^{*}(x)= \begin{cases}y_{+}(x) & x \in\left\{z: \Re(z) \leq x_{2}, \Im\left(D_{2}\left(z_{++}\right)\right)<0\right\} \cup\left(-\infty, x_{1}\right), \\ y_{+}(x) & x \in\left\{z: \Re(z) \geq x_{3}, \Im\left(D_{2}\left(z_{++}\right)\right)>0\right\} \cup\left(x_{4}, \infty\right), \\ y_{-}(x) & \text { otherwise }\end{cases}
$$

is an analytic function in $\mathbb{C} \backslash\left(\left[x_{1}, x_{2}\right] \cup\left[x_{3}, x_{4}\right]\right)$.

Proof. Let $x=u+i v$ with $u, v \in \mathbb{R}$. We write $D_{2}(x)=\Re\left(D_{2}(x)\right)+i \Im\left(D_{2}(x)\right)$. Since

$$
D_{2}(x)=\nu^{2}(x)-4 \mu(x) \delta(x) .
$$

Using $x=u+i v$ to rewrite the functions $\nu(),. \mu(),. \delta($.$) as$

$$
\begin{aligned}
\left.\nu(x)\right|_{x=u+i v} & =r_{2} \tilde{s}_{2} \xi_{1}(u+i v)^{2}+\left(\tilde{r}_{1} \xi_{2}+r_{2} \tilde{s}_{2} r_{1} \tilde{s}_{1}+\tilde{r}_{2} \xi_{1}-1\right)(u+i v)+\tilde{r}_{2} r_{1} \tilde{s}_{1} \\
& =\left[r_{2} \tilde{s}_{2} \xi_{1}\left(u^{2}+v^{2}\right)+\left(\tilde{r}_{1} \xi_{2}+r_{2} \tilde{s}_{2} r_{1} \tilde{s}_{1}+\tilde{r}_{2} \xi_{1}-1\right) u+\tilde{r}_{2} r_{1} \tilde{s}_{1}\right] \\
& +i\left[2 r_{2} \tilde{s}_{2} \xi_{1} u v+\left(\tilde{r}_{1} \xi_{2}+r_{2} \tilde{s}_{2} r_{1} \tilde{s}_{1}+\tilde{r}_{2} \xi_{1}-1\right) v\right] \\
\mu & \\
\mu & =\left(\xi_{1} \xi_{2}\left(u^{2}-v^{2}\right)+r_{1} \tilde{s}_{1} \xi_{2} u\right)+\left(2 \xi_{1} \xi_{2} u v+r_{1} \tilde{s}_{1} \xi_{2} v\right) i
\end{aligned}
$$

and

$$
\begin{aligned}
\left.\delta(x)\right|_{x=u+i v} & =\tilde{r}_{1} r_{2} \tilde{s}_{2}(u+i v)+\tilde{r}_{1} \tilde{r}_{2} \\
& =\left(\tilde{r}_{1} r_{2} \tilde{s}_{2} u+\tilde{r}_{1} \tilde{r}_{2}\right)+\left(\tilde{r}_{1} r_{2} \tilde{s}_{2} v\right) i .
\end{aligned}
$$

Now we can write $D_{2}(x)$ as

$$
\begin{aligned}
\left.D_{2}(x)\right|_{x=u+i v} & =\nu(u, v)^{2}-4 \mu(u, v) \delta(u, v) \\
& =\left[r_{2} \tilde{s}_{2} \xi_{1}\left(u^{2}+v^{2}\right)+\left(\tilde{r}_{1} \xi_{2}+r_{2} \tilde{s}_{2} r_{1} \tilde{s}_{1}+\tilde{r}_{2} \xi_{1}-1\right) u+\tilde{r}_{2} r_{1} \tilde{s}_{1}\right]^{2} \\
& -\left[2 r_{2} \tilde{s}_{2} \xi_{1} u v+\left(\tilde{r}_{1} \xi_{2}+r_{2} \tilde{s}_{2} r_{1} \tilde{s}_{1}+\tilde{r}_{2} \xi_{1}-1\right) v\right]^{2} \\
& +2 i\left[r_{2} \tilde{s}_{2} \xi_{1}\left(u^{2}+v^{2}\right)+\left(\tilde{r}_{1} \xi_{2}+r_{2} \tilde{s}_{2} r_{1} \tilde{s}_{1}+\tilde{r}_{2} \xi_{1}-1\right) u+\tilde{r}_{2} r_{1} \tilde{s}_{1}\right] \times \\
& {\left[2 r_{2} \tilde{s}_{2} \xi_{1} u v+\left(\tilde{r}_{1} \xi_{2}+r_{2} \tilde{s}_{2} r_{1} \tilde{s}_{1}+\tilde{r}_{2} \xi_{1}-1\right) v\right] } \\
- & 4\left[\left(\xi_{1} \xi_{2}\left(u^{2}-v^{2}\right)+r_{1} \tilde{s}_{1} \xi_{2} u\right)+i\left(2 \xi_{1} \xi_{2} u v+r_{1} \tilde{s}_{1} \xi_{2} v\right)\right]\left[\left(\tilde{r}_{1} r_{2} \tilde{s}_{2} u+\tilde{r}_{1} \tilde{r}_{2}\right)+i \tilde{r}_{1} r_{2} \tilde{s}_{2} v\right] .
\end{aligned}
$$


Using the above equation we can write the real and imaginary parts of $D_{2}(x)$ as follows

$$
\begin{aligned}
\Re\left(D_{2}(x)\right) & =\left[r_{2} \tilde{s}_{2} \xi_{1}\left(u^{2}+v^{2}\right)+\left(\tilde{r}_{1} \xi_{2}+r_{2} \tilde{s}_{2} r_{1} \tilde{s}_{1}+\tilde{r}_{2} \xi_{1}-1\right) u+\tilde{r}_{2} r_{1} \tilde{s}_{1}\right]^{2} \\
& -\left[2 r_{2} \tilde{s}_{2} \xi_{1} u v+\left(\tilde{r}_{1} \xi_{2}+r_{2} \tilde{s}_{2} r_{1} \tilde{s}_{1}+\tilde{r}_{2} \xi_{1}-1\right) v\right]^{2} \\
& -4\left(\xi_{1} \xi_{2}\left(u^{2}-v^{2}\right)+r_{1} \tilde{s}_{1} \xi_{2} u\right)\left(\tilde{r}_{1} r_{2} \tilde{s}_{2} u+\tilde{r}_{1} \tilde{r}_{2}\right)+4\left(2 \xi_{1} \xi_{2} u v+r_{1} \tilde{s}_{1} \xi_{2} v\right) \tilde{r}_{1} r_{2} \tilde{s}_{2} v
\end{aligned}
$$

and

$$
\begin{aligned}
\Im\left(D_{2}(x)\right) & =2\left[r_{2} \tilde{s}_{2} \xi_{1}\left(u^{2}+v^{2}\right)+\left(\tilde{r}_{1} \xi_{2}+r_{2} \tilde{s}_{2} r_{1} \tilde{s}_{1}+\tilde{r}_{2} \xi_{1}-1\right) u+\tilde{r}_{2} r_{1} \tilde{s}_{1}\right] \times \\
& {\left[2 r_{2} \tilde{s}_{2} \xi_{1} u v+\left(\tilde{r}_{1} \xi_{2}+r_{2} \tilde{s}_{2} r_{1} \tilde{s}_{1}+\tilde{r}_{2} \xi_{1}-1\right) v\right] } \\
& -4\left(\xi_{1} \xi_{2}\left(u^{2}-v^{2}\right)+r_{1} \tilde{s}_{1} \xi_{2} u\right) \tilde{r}_{1} r_{2} \tilde{s}_{2} v-4\left(2 \xi_{1} \xi_{2} u v+r_{1} \tilde{s}_{1} \xi_{2} v\right)\left(\tilde{r}_{1} r_{2} \tilde{s}_{2} u+\tilde{r}_{1} \tilde{r}_{2}\right) .
\end{aligned}
$$

It is obvious from $(25)$ that $\Im\left(D_{2}(x)\right)=0$ if $(u, v)$ satisfies

$$
\begin{aligned}
& {\left[r_{2} \tilde{s}_{2} \xi_{1}\left(u^{2}+v^{2}\right)+\left(\tilde{r}_{1} \xi_{2}+r_{2} \tilde{s}_{2} r_{1} \tilde{s}_{1}+\tilde{r}_{2} \xi_{1}-1\right) u+\tilde{r}_{2} r_{1} \tilde{s}_{1}\right]} \\
& \times v\left[2 r_{2} \tilde{s}_{2} \xi_{1} u+\tilde{r}_{1} \xi_{2}+r_{2} \tilde{s}_{2} r_{1} \tilde{s}_{1}+\tilde{r}_{2} \xi_{1}-1\right] \\
& =2\left(\xi_{1} \xi_{2}\left(u^{2}-v^{2}\right)+r_{1} \tilde{s}_{1} \xi_{2} u\right) \tilde{r}_{1} r_{2} \tilde{s}_{2} v-2\left(2 \xi_{1} \xi_{2} u v+r_{1} \tilde{s}_{1} \xi_{2} v\right)\left(\tilde{r}_{1} r_{2} \tilde{s}_{2} u+\tilde{r}_{1} \tilde{r}_{2}\right),
\end{aligned}
$$

which can be written as

$$
\begin{aligned}
& {\left[2 r_{2} \tilde{s}_{2} \xi_{1} r_{2} \tilde{s}_{2} \xi_{1} u+r_{2} \tilde{s}_{2} \xi_{1} \tilde{r}_{1} \xi_{2}+r_{2} \tilde{s}_{2} \xi_{1} r_{2} \tilde{s}_{2} r_{1} \tilde{s}_{1}+r_{2} \tilde{s}_{2} \xi_{1} \tilde{r}_{2} \xi_{1}-r_{2} \tilde{s}_{2} \xi_{1}+2 \xi_{1} \xi_{2} \tilde{r}_{1} r_{2} \tilde{s}_{2}\right] v^{2} } \\
= & -2 r_{2} \tilde{s}_{2} \xi_{1} r_{2} \tilde{s}_{2} \xi_{1} u^{3}-\left[r_{2} \tilde{s}_{2} \xi_{1} \tilde{r}_{1} \xi_{2}+r_{2} \tilde{s}_{2} \xi_{1} r_{2} \tilde{s}_{2} r_{1} \tilde{s}_{1}+r_{2} \tilde{s}_{2} \xi_{1} \tilde{r}_{2} \xi_{1}-r_{2} \tilde{s}_{2} \xi_{1}\right] u^{2} \\
+ & 2 \xi_{1} \xi_{2} \tilde{r}_{1} r_{2} \tilde{s}_{2} u^{2}-4 \xi_{1} \xi_{2} \tilde{r}_{1} r_{2} \tilde{s}_{2} u^{2}-2 r_{2} \tilde{s}_{2} \xi_{1} u^{2}\left(\tilde{r}_{1} \xi_{2}+r_{2} \tilde{s}_{2} r_{1} \tilde{s}_{1}+\tilde{r}_{2} \xi_{1}-1\right) \\
& +2 r_{1} \tilde{s}_{1} \xi_{2} \tilde{r}_{1} r_{2} \tilde{s}_{2} u-\tilde{r}_{1} \xi_{2} u\left(\tilde{r}_{1} \xi_{2}+r_{2} \tilde{s}_{2} r_{1} \tilde{s}_{1}+\tilde{r}_{2} \xi_{1}-1\right) \\
& -r_{2} \tilde{s}_{2} r_{1} \tilde{s}_{1} u\left(\tilde{r}_{1} \xi_{2}+r_{2} \tilde{s}_{2} r_{1} \tilde{s}_{1}+\tilde{r}_{2} \xi_{1}-1\right)-\tilde{r}_{2} \xi_{1} u\left(\tilde{r}_{1} \xi_{2}+r_{2} \tilde{s}_{2} r_{1} \tilde{s}_{1}+\tilde{r}_{2} \xi_{1}-1\right) \\
& +u\left(\tilde{r}_{1} \xi_{2}+r_{2} \tilde{s}_{2} r_{1} \tilde{s}_{1}+\tilde{r}_{2} \xi_{1}-1\right)-2 r_{2} \tilde{s}_{2} \xi_{1} \tilde{r}_{2} r_{1} \tilde{s}_{1} u-4 \xi_{1} \xi_{2} \tilde{r}_{1} \tilde{r}_{2} u-2 r_{1} \tilde{s}_{1} \xi_{2} \tilde{r}_{1} r_{2} \tilde{s}_{2} u \\
& -\tilde{r}_{1} \xi_{2} \tilde{r}_{2} r_{1} \tilde{s}_{1}-r_{2} \tilde{s}_{2} r_{1} \tilde{s}_{1} \tilde{r}_{2} r_{1} \tilde{s}_{1}-2 r_{1} \tilde{s}_{1} \xi_{2} \tilde{r}_{1} \tilde{r}_{2}-\tilde{r}_{2} \xi_{1} \tilde{r}_{2} r_{1} \tilde{s}_{1}+\tilde{r}_{2} r_{1} \tilde{s}_{1} .
\end{aligned}
$$

For simplicity reasons the above equation can be written in the form

$$
\left(a_{1} u+a_{2}\right) v^{2}=a_{3} u^{3}+a_{4} u^{2}+a_{5} u+a_{6},
$$

where

$$
\begin{gathered}
a_{1}=-2 r_{2}^{2} \tilde{s}_{2}^{2} \xi_{1}^{2}<0 \\
a_{2}=-3 r_{2} \tilde{s}_{2} \tilde{r}_{1} \xi_{1} \xi_{2}-r_{2}^{2} \tilde{s}_{2}^{2} r_{1} \tilde{s}_{1} \xi_{1}-r_{2} \tilde{s}_{2} \tilde{r}_{2} \xi_{1}^{2}+r_{2} \tilde{s}_{2} \xi_{1}>0, \\
a_{3}=2 r_{2}^{2} \tilde{s}_{2}^{2} \xi_{1}^{2}>0 \\
a_{4}=3 \tilde{r}_{1} r_{2} \tilde{s}_{2} \xi_{1} \xi_{2}+r_{2}^{2} \tilde{s}_{2}^{2} r_{1} \tilde{s}_{1} \xi_{1}+r_{2} \tilde{s}_{2} \tilde{r}_{2} \xi_{1}^{2}-r_{2} \tilde{s}_{2} \xi_{1} \\
+2 r_{2} \tilde{s}_{2} \xi_{1}\left(\tilde{r}_{1} \xi_{2}+r_{2} \tilde{s}_{2} r_{1} \tilde{s}_{1}+\tilde{r}_{2} \xi_{1}-1\right)<0, \\
a_{5}=-2 r_{1} \tilde{s}_{1} \xi_{2} \tilde{r}_{1} r_{2} \tilde{s}_{2}+\tilde{r}_{1} \xi_{2}\left(\tilde{r}_{1} \xi_{2}+r_{2} \tilde{s}_{2} r_{1} \tilde{s}_{1}+\tilde{r}_{2} \xi_{1}-1\right) \\
+r_{2} \tilde{s}_{2} r_{1} \tilde{s}_{1}\left(\tilde{r}_{1} \xi_{2}+r_{2} \tilde{s}_{2} r_{1} \tilde{s}_{1}+\tilde{r}_{2} \xi_{1}-1\right)+\tilde{r}_{2} \xi_{1}\left(\tilde{r}_{1} \xi_{2}+r_{2} \tilde{s}_{2} r_{1} \tilde{s}_{1}+\tilde{r}_{2} \xi_{1}-1\right) \\
-\left(\tilde{r}_{1} \xi_{2}+r_{2} \tilde{s}_{2} r_{1} \tilde{s}_{1}+\tilde{r}_{2} \xi_{1}-1\right)+2 r_{2} \tilde{s}_{2} \xi_{1} \tilde{r}_{2} r_{1} \tilde{s}_{1} \\
+4 \xi_{1} \xi_{2} \tilde{r}_{1} \tilde{r}_{2}+2 r_{1} \tilde{s}_{1} \xi_{2} \tilde{r}_{1} r_{2} \tilde{s}_{2}>0,
\end{gathered}
$$

and

$$
a_{6}=3 \tilde{r}_{1} \xi_{2} \tilde{r}_{2} r_{1} \tilde{s}_{1}+r_{2} \tilde{s}_{2} r_{1}^{2} \tilde{s}_{1} \tilde{r}_{2} \tilde{s}_{1}^{2}+\tilde{r}_{2}^{2} \xi_{1} r_{1}-\tilde{r}_{2} r_{1} \tilde{s}_{1}>0 .
$$

It should be noted that (29-34) satisfied by the coefficients $a_{j}, j=1, \cdots, 6$ are tested using some stability system parameters i.e. parameters satisfying $\left(\xi_{1}<1-r_{2}\right.$ and $\left.\xi_{2}<1-r_{1}\right)$. Assume that $d_{1}(u)$ is the polynomial in the right-hand side of (28). 
It is easy to see that $\lim _{u \rightarrow+\infty} d_{1}(u)>0$, and $d_{1}(0)>0$. The polynomial $d_{1}$ is of degree three and has at least one real root. Equation (28) has two roots in $v$ given by

$$
\begin{aligned}
v & = \pm \sqrt{\frac{a_{3} u^{3}+a_{4} u^{2}+a_{5} u+a_{6}}{a_{1} u+a_{2}}} \\
& = \pm \sqrt{\frac{d_{1}(u)}{a_{1} u+a_{2}}} .
\end{aligned}
$$

From (35) we would then obtain two curves, one in the half-plane $\{x: \Im(x)>0\}$ (corresponding to the plus sign) and the other in the half-plane $\{x: \Im(x)<0\}$ (corresponding to the minus sign). Along each of these curves, the sign of $\Re\left(D_{2}(x)\right)$ should be constant since the imaginary and real parts cancel only for $x \in \mathbb{R}$ (namely for $x$ equals to one of the roots $x_{1}, x_{2}, x_{3}$ and $\left.x_{4}\right)$. When $u \rightarrow-\infty, v^{2} \sim-u^{2}$ and then $\Re\left(D_{2}(x)\right) \sim-u^{3}$, which contradicts the fact that the sign of $\Re\left(D_{2}(x)\right)$ should be constant along the curve $\Im\left(D_{2}(x)\right)=0$. As a consequence, the polynomial $d_{1}(u)$ has three real roots ( $\left.\operatorname{say} x_{1 a}, x_{2 a}, x_{3 a}\right)$, which are positive since $d_{1}(0)>0$ such that $x_{1 a} \in(0,1)$ and $x_{2 a}, x_{3 a} \in(1, \infty)$ we assume that

$$
0<x_{1} \leq x_{1 a}<x_{2}<1<x_{2 a} \leq x_{3} \leq x_{3 a} \leq x_{4} .
$$

In order to prove that the function $Y^{*}(x)$ is analytic in the whole of $\mathbb{C}$ deprived of the segments $\left(\left[x_{1}, x_{2}\right] \cup\left[x_{3}, x_{4}\right]\right)$, by Moreira's theorem (see e.g. [17,18]), it is sufficient to show that this function is continuous on the branch $\left\{x: \Im\left(D_{2}(x)\right)=\right.$ $\left.0, \Re\left(D_{2}(x)\right) \leq 0\right\}$ separating the two above domains. But this is clearly checked from the choice of the determination of the square root.

This lemma will be used in the reduction to the boundary value problem.

\section{Potential singularities of the unknowns}

In this section we will use the idea of the resultant between two polynomials to investigate the potential singularities of the unknowns.

\subsection{The main idea of the singularities}

Generally speaking, when we have two polynomials in two variables, say,

$$
g_{1}(x, y)=a_{0}(y)+a_{1}(y) x+\cdots+a_{n_{1}}(y) x^{n_{1}},
$$

and

$$
g_{2}(x, y)=b_{0}(y)+b_{1}(y) x+\cdots+b_{n_{2}}(y) x^{n_{2}},
$$

the resultant $\left(\operatorname{Res}_{x}\left(g_{1}, g_{2} ; y\right)\right)$ (see e.g. [32] in Appendix B) of the polynomials $g_{1}$ and $g_{2}$ with respect to $x$ is the determinant of the matrix

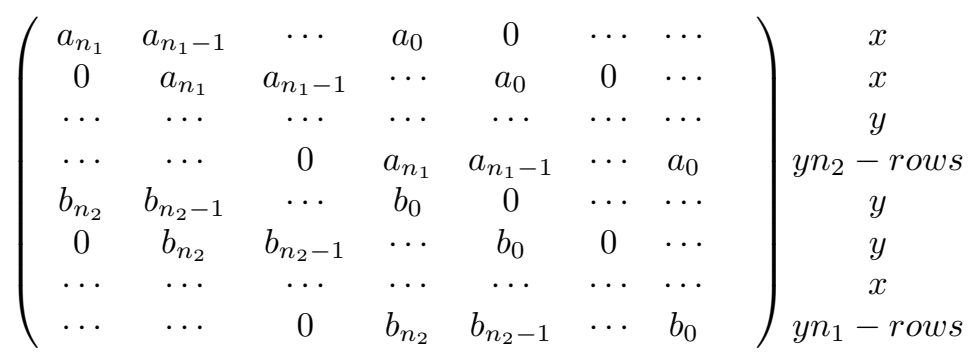

which is a polynomial in $y$. The resultant with respect to $x$ is 0 at $y_{0}$ if the polynomials $g_{1}$ and $g_{2}$ have a common nontrivial root $\left(x_{0}, y_{0}\right)$ or the leading coefficients are zero. We used the results published in [33].

\section{Boundary value problem}

In this section we will describe how our functional equation could be solved by reduction to the Riemann-Hilbert boundary value problem via using some conformal mapping. 


\subsection{The unknown $F(x)$}

The function $x_{ \pm}(y)$ defined by (14) is defined in $\mathbb{C} \backslash\left(\left[y_{1}, y_{2}\right] \cup\left[y_{3}, y_{4}\right]\right)$. Assume now that the image of the slit $\left[y_{1}, y_{2}\right]$ by $x_{ \pm}(y)$ is a closed contour $L_{1}$ see figure 1 . The following Lemmas show how the functional equation (9) is reduced to a Riemmann-Hilbert boundary value problem in the unknown $F(x)$. Regarding the analyticity of the function $F$ in $L_{1}^{+}$since we cannot guarantee that such function is analytic in $L_{1}^{+}$then we have to consider two cases:

Case 1: If the function $F(x)$ is analytic in $L_{1}^{+}$.

In this case the main functional equation is reduced to the following Riemann-Hilbert Boundary value problem.

Lemma 6.1. Find an analytic function $F$ such that

$$
\Re\left(\mathrm{i}_{1}(\tau(u)) F(\tau(u))\right)=\Re\left(m_{2}(\tau(u))\right), u \in D
$$

where $\tau(u)$ is the inverse of some conformal mapping, and $m_{1}(u)$ and $m_{2}(u)$ are known functions.

Proof. Since the main PGF function $f(x, y)$ is analytic in the unit disk, this implies that if $A_{1}(x, y)=0$ then also

$$
A_{2}(x, y) f(x, 0)+A_{3}(x, y) f(0, y)+A_{4}(x, y) f(0,0)=0,
$$

which is equivalent to

$$
A_{2}(x, y) F(x)+A_{3}(x, y) G(y)+A_{4}(x, y)=0 .
$$

Now assume that $y \in\left(y_{1}, y_{2}\right)$ then $y \in \mathbb{R}, x \in L_{1}$, so $\Re(i(G(y)))=0$. Now equation (37) can be written as follows

$$
\Re\left(\mathrm{i} \frac{A_{2}(x, y)}{A_{3}(x, y)} F(x)\right)=-\Re\left(\mathrm{i} \frac{A_{4}(x, y)}{A_{3}(x, y)}\right),
$$

the right hand side of the above equation can be written as follows

$$
-\Re\left(\mathrm{i} \frac{A_{4}(x, y)}{A_{3}(x, y)}\right)=\Im\left(\frac{A_{4}(x, y)}{A_{3}(x, y)}\right) .
$$

Now for $x \in L_{1}$ and $y=Y^{*}(x)$, we have a problem of the form

$$
\Re\left(\mathrm{i} \frac{A_{2}\left(x, Y^{*}(x)\right)}{A_{3}\left(x, Y^{*}(x)\right)} F(x)\right)=\Im\left(\frac{A_{4}\left(x, Y^{*}(x)\right)}{A_{3}\left(x, Y^{*}(x)\right)}\right),
$$

which is a Riemann-Hilbert boundary value problem. To solve the constructed Riemann-Hilbert problem, a classical approach (see e.g [21]) is to consider a conformal mapping $\theta$ between the bounded domain $L_{1}^{+}$delineated by the contour $L_{1}$ and the unit disk

$$
\theta_{x}(x): L_{1}^{+} \rightarrow D^{+}
$$

with inverse

$$
\tau_{x}(u): D^{+} \rightarrow L_{1}^{+} .
$$

This conformal mapping exists by Riemann's conformal mapping theorem. The function $\theta($.$) can be chosen so as to preserve$ the symmetry with respect to the horizontal axis. The Riemann-Hilbert problem (38) is then transformed into a RiemannHilbert problem of the form

$$
\Re\left(\mathrm{i} m_{1}(\tau(u)) F(\tau(u))\right)=\Re\left(m_{2}(\tau(u))\right),
$$

for some functions $m_{1}(u)$ and $m_{2}(u)$ given by

$$
m_{1}(\tau(u))=\frac{C_{2}\left(\tau_{x}(u), Y^{*}\left(\tau_{x}(u)\right)\right)}{C_{3}\left(\tau_{x}(u), Y^{*}\left(\tau_{x}(u)\right)\right)},
$$

and

$$
m_{2}(\tau(u))=\frac{C_{4}\left(\tau_{x}(u), Y^{*}\left(\tau_{x}(u)\right)\right)}{C_{3}\left(\tau_{x}(u), Y^{*}\left(\tau_{x}(u)\right)\right)} .
$$


An explicit form of this conformal mapping is not an easy task but can be obtained using the results in e.g. [35-37]. Case 2: If the function $F(x)$ has potential singularities in $L_{1}^{+}$

In this case we will remove such potential singularities and the main functional equation is reduced to the following boundary value problem.

Lemma 6.2. Find an analytic function $F_{x}\left(\Omega_{x}(u)\right)$ satisfying

$$
\begin{aligned}
& \Re\left(\mathrm{i} \frac{A_{2}\left(\Omega_{x}(u), Y^{*}\left(\Omega_{x}(u)\right)\right)}{A_{3}\left(\Omega_{x}(u), Y^{*}\left(\Omega_{x}(u)\right)\right)} F_{x}\left(\Omega_{x}(u)\right)\right)= \\
& \Im\left[\frac{A_{4}\left(\Omega_{x}(u), Y^{*}\left(\Omega_{x}(u)\right)\right)}{A_{3}\left(\Omega_{x}(u), Y^{*}\left(\Omega_{x}(u)\right)\right)}+\frac{A_{2}\left(\Omega_{x}(u), Y^{*}\left(\Omega_{x}(u)\right)\right)}{A_{3}\left(\Omega_{x}(u), Y^{*}\left(\Omega_{x}(u)\right)\right)} \frac{R_{x}}{\Omega_{x}(u)-x^{*}}\right], u \in D
\end{aligned}
$$

which is a special case of (1).

Proof. Since the main PGF function $f(x, y)$ is analytic in the unit disk, this implies that if $C_{1}(x, y)=0$ then also

$$
A_{2}(x, y) f(x, 0)+A_{3}(x, y) f(0, y)+A_{4}(x, y) f(0,0)=0,
$$

which is equivalent to

$$
A_{2}(x, y) F(x)+A_{3}(x, y) G(y)+A_{4}(x, y)=0 .
$$

Now assume that $y \in\left(y_{1}, y_{2}\right)$ then $y \in \mathbb{R}$, so $\Re(i(G(y)))=0$, now equation (40) can be written as follows

$$
\Re\left(\mathrm{i} \frac{A_{2}(x, y)}{A_{3}(x, y)} F(x)\right)=-\Re\left(\mathrm{i} \frac{A_{4}(x, y)}{A_{3}(x, y)}\right),
$$

the right hand side of the above equation can be written as follows

$$
-\Re\left(\mathrm{i} \frac{A_{4}(x, y)}{A_{3}(x, y)}\right)=\Im\left(\frac{A_{4}(x, y)}{A_{3}(x, y)}\right) .
$$

Now for $x \in L_{1}$ and $y=Y^{*}(x)$, we have a problem of the form

$$
\Re\left(\mathrm{i} \frac{A_{2}\left(x, Y^{*}(x)\right)}{A_{3}\left(x, Y^{*}(x)\right)} F(x)\right)=\Im\left(\frac{A_{4}\left(x, Y^{*}(x)\right)}{A_{3}\left(x, Y^{*}(x)\right)}\right) .
$$

Since the function $F(x)$ has potential singularities inside the contour $L_{1}$, then in order to remove such singularities we assume that

$$
F_{x}(x):=F(x)-\frac{R_{x}}{x-x^{*}},
$$

where $x^{*}$ represents the potential singularities of $F(x)$ that lies inside the contour $L_{1}$ with residue $R_{x}$. Using (41), the function $F_{x}(x)$ is an analytic function in the interior of the contour $L_{1}$ and satisfies for $x \in L_{1}$ and $y=Y^{*}(x)$

$$
\Re\left(\mathrm{i} \frac{A_{2}\left(x, Y^{*}(x)\right)}{A_{3}\left(x, Y^{*}(x)\right)}\left[F_{x}(x)+\frac{R_{x}}{x-x^{*}}\right]\right)=\Im\left(\frac{A_{4}\left(x, Y^{*}(x)\right)}{A_{3}\left(x, Y^{*}(x)\right)}\right) .
$$

The residue $R_{x}$ of the function $F(x)$ at $x^{*}$ is given by

$$
R_{x}=-\frac{A_{3}\left(x^{*}, y^{*}\right) G\left(y^{*}\right)+A_{4}\left(x^{*}, y^{*}\right)}{\frac{\partial}{\partial x} A_{2}\left(x^{*}, y^{*}\right)+\frac{\partial}{\partial y} A_{2}\left(x^{*}, y^{*}\right) \frac{d Y^{*}}{d x}\left(x^{*}\right)},
$$

where $y^{*}=Y^{*}\left(x^{*}\right)$. It should also be noted that from section 5 , the function $F(x)$ has simple poles. In the case that there are more than one simple pole singularities then (42) will be modified to be

$$
F_{x}(x):=F(x)-\sum_{k=1}^{n^{\sharp}} \frac{R_{x}}{x-x_{k}},
$$


where $n^{\sharp}$ is the number of singularities that lie inside the contour $L_{1}$. If this is the case then (43) will be changed a bit. To solve the problem (43), we consider the conformal mapping $\Lambda_{x}(x)$ from $L_{1}^{+}$onto the unit disk

$$
\Lambda_{x}(x): L_{1}^{+} \rightarrow D^{+}
$$

with inverse

$$
\Omega_{x}(u): D^{+} \rightarrow L_{1}^{+} .
$$

This conformal mapping can be chosen to preserve the symmetry with respect to the horizontal axis, because in this case the real axis is an axis of symmetry. Moreover, by imposing the condition $\Lambda_{x}(0)=0$, the conformal mapping $\Lambda_{x}(x)$ is unique. We are then led to consider the following problem on the unit circle: The function $F_{x}\left(\Omega_{x}(u)\right)$ is analytic in the unit disk $D^{+}$ and satisfies for $u$ on the unit circle $D$

$$
\begin{aligned}
\Re\left(\mathrm{i} \frac{A_{2}\left(\Omega_{x}(u), Y^{*}\left(\Omega_{x}(u)\right)\right)}{A_{3}\left(\Omega_{x}(u), Y^{*}\left(\Omega_{x}(u)\right)\right)} F_{x}\left(\Omega_{x}(u)\right)\right) & =\Im\left(\frac{A_{4}\left(\Omega_{x}(u), Y^{*}\left(\Omega_{x}(u)\right)\right)}{A_{3}\left(\Omega_{x}(u), Y^{*}\left(\Omega_{x}(u)\right)\right)}\right) \\
& -\Re\left(\mathrm{i} \frac{A_{2}\left(\Omega_{x}(u), Y^{*}\left(\Omega_{x}(u)\right)\right)}{A_{3}\left(\Omega_{x}(u), Y^{*}\left(\Omega_{x}(u)\right)\right)} \frac{R_{x}}{\Omega_{x}(u)-x^{*}} .\right.
\end{aligned}
$$

\subsection{The solutions}

The unknown $F(x)$ is given as a solution of the boundary value problem constructed in the previous lemma

$$
\begin{aligned}
F(x) & :=\frac{f(x, 0)}{f(0,0)}=F_{x}(x)+\frac{R_{x}}{x-x^{*}} \\
& =\frac{R_{x}}{x-x^{*}}+\frac{\phi^{i}(x)}{2 \pi i} \int_{L_{1}} \frac{A_{x}(z) d z}{\phi^{i}(z)(z-x)}+Q(x) \phi(x),
\end{aligned}
$$

where $Q(x)$ is a polynomial, which can be determined by using the conditions at infinity, the function $A(x)$ is given by

$$
A_{x}(u)=-2 \mathrm{i} \frac{b_{x}(u)}{a_{x}(u)}
$$

and the function $\phi($.$) is given as in the introduction section:$

$$
\phi(u)= \begin{cases}\exp \left(\frac{1}{2 i \pi} \int_{L_{1}} \log \left(z^{-\kappa} \frac{\overline{a(z)}}{a(z)}\right) \frac{d z}{z-u}\right) & \text { if } u \in L_{1}^{+} \\ \frac{1}{u^{\kappa}} \exp \left(\frac{1}{2 i \pi} \int_{L_{1}} \log \left(z^{-\kappa} \frac{\overline{a(z)}}{a(z)}\right) \frac{d z}{z-u}\right) & \text { if } u \in L_{1}^{-}\end{cases}
$$

In the current case we have

$$
a_{x}(u)=\frac{A_{2}\left(\Omega_{x}(u), Y^{*}\left(\Omega_{x}(u)\right)\right)}{A_{3}\left(\Omega_{x}(u), Y^{*}\left(\Omega_{x}(u)\right)\right)},
$$

and

$$
b_{x}(u)=\Im\left[\frac{A_{4}\left(\Omega_{x}(u), Y^{*}\left(\Omega_{x}(u)\right)\right)}{A_{3}\left(\Omega_{x}(u), Y^{*}\left(\Omega_{x}(u)\right)\right)}+\frac{A_{2}\left(\Omega_{x}(u), Y^{*}\left(\Omega_{x}(u)\right)\right)}{A_{3}\left(\Omega_{x}(u), Y^{*}\left(\Omega_{x}(u)\right)\right)} \frac{R_{x}}{\Omega_{x}(u)-x^{*}}\right] .
$$

The index $(\kappa)$ of this Riemann-Hilbert boundary value problem is defined as the increment of the argument of the function $\overline{a_{x}(u)} / a_{x}(u)$ divided by $2 \pi$ when $u$ traverses the unit circle once. It should be noted that according to [20] and based on the value of the index $\kappa$ we have the following:

1. if $\kappa=0$,

there exists a unique solution of the problem corresponding to $Q(x) \equiv 0$;

2. if $\kappa>0$,

there exists an infinite number of solutions of the problem corresponding to each polynomial $Q(x)$ of degree less than or equal to $\kappa-1$. 
3. if $\kappa<0$,

there exists a unique solution if and only if the function $A(z)$ satisfies the conditions:

$$
\int_{L} \frac{\lambda^{m} A(\lambda) d \lambda}{\phi_{i}(\lambda)}=0, \quad 0 \leq m \leq-(\kappa+1)
$$

The unknown $f(0,0)$ can be eventually obtained using $(44)$, and using $f(1,0)=\left(\xi_{2}-\tilde{r_{1}}\right) / \tilde{r_{1}}$ which is obtained by using the normalization condition $f(1,1)=1$ in the original equation.

\section{Conclusion}

In this article we investigated the solution of a challenging two-place functional equation which arose from a queueing model of a network gateway. The solution is obtained by the following steps: First, an extensive analysis of the kernel is done. Second, an analytic continuation of the function defined by the kernel is proved. Third, the potential singularities of the unknowns are obtained directly from the idea of the resultant. The last step is the reduction to boundary value problem using some conformal mapping. It should be clarified that the solution is only given as a function of some conformal mapping between $L_{1}^{+}$and the unit disk. According to some recent work an explicit form of such conformal mapping exists only for a certain type of random walks which is not the case in our case. The conformal mappings in those recent cases are obtained using the conformal gluing function process. However, for a general random walk such a conformal gluing process exist and can be obtained, which will be some potential future work.

\section{Acknowledgements}

We are grateful to the Referees for a very careful and helpful reading of the paper, freeing it of potential ambiguities, and for valuable comments.

\section{References}

[1] J. Aczél, Functional Equations: History, Applications and Theory, Springer Science \& Business Media, vol. 12.(2012)

[2] S. Czerwik, Functional equations and inequalities in several variables, World Scientific Publishing Co., Inc., River Edge, NJ, (2002).

[3] G. Maksa, The general solution of a functional equation related to the mixed theory of information, Aequationes Mathematicae, Springer, vol. 22, no. 1, (1981) pp. 90-96.

[4] E. Castillo, A. Iglesias, R. Ruz-Cobo, Functional equations in applied sciences, Mathematics in Science and Engineering,Vol. 199. Elsevier, (2004).

[5] El-S. El-hady, W, Frg-Rob, and M. Mahmoud, On a two-variable functional equation arising from databases, WSEAS Trans. Math, vol. 14, (2015) pp. 265-270.

[6] J.W. Cohen, On the asymmetric clocked buffered switch, Queueing Syst., vol. 30, no. 3-4, (1998) pp. 385-404.

[7] EL-S. El-Hady, J. Brzdek, W. Förg-Rob, H. Nassar, Remarks on Solutions of a Functional Equation Arising from an Asymmetric Switch, Contributions in Mathematics and Engineering: In Honor of Constantin Carathéodory, (2016) pp. $153-163$

[8] H. Nassar, Two-dimensional queueing model for a LAN gateway, WSEAS PRESS, vol. 5, no. 9, (2006).

[9] EL-S. El-Hady, J. Brzdek, H. Nassar, On the structure and solutions of functional equations arising from queueing models, Aequationes mathematicae, doi: 10.1007/s00010-017-0471-1, (2017) pp. 1-33.

[10] H. Nassar and EL-S. El-Hady, Closed-Form Solution of a LAN Gateway Queueing Model, Contributions in Mathematics and Engineering: In Honor of Constantin Carathéodory, (2016) pp. 393-427. 
[11] V.A. Malyshev, An analytical method in the theory of two-dimensional positive random walks, Siberian Mathematical Journal, vol. 13, no. 6, (1972) pp.917-929.

[12] G. Fayolle and R. Iasnogorodski, Two coupled processors: the reduction to a Riemann-Hilbert problem, Zeitschrift für Wahrscheinlichkeitstheorie und verwandte Gebiete, vol. 47, no. 3, (1979). pp. 325-351.

[13] J. W. Cohen, O. Boxma, Boundary value problems in queueing system analysis, vol. 79, Elsevier.(2000)

[14] G. Fayolle, R. Iasnogorodski, V.A. Malyshev, Random walks in the quarter-plane: algebraic methods, boundary value problems and applications, vol. 40, Springer.(1999)

[15] S. Ponnusamy, An introduction to complex analysis, 1st edition, Springer. (2011)

[16] S. Ponnusamy, Applied and computational complex analysis, discrete Fourier analysis, Cauchy integrals, construction of conformal maps, univalent functions, John Wiley \& Sons, vol. 3. (1993)

[17] S. Ponnusamy, Complex analysis an introduction to the Theory of Analytic Functions of One Complex Variable, McGrawHill Book Company, vol. 3. (1966)

[18] S. Ponnusamy, Complex variables with applications, Birkhäuser, Boston.(2006)

[19] F. D. Gakhov, Boundary value problems, Courier Corporation.(1990)

[20] S. Ponnusamy, Mathematical analysis and numerical methods for science and technology, vol. 4. (1991)

[21] J. Resing, and L. Örmeci, A tandem queueing model with coupled processors, Operations Research Letters, vol.31, no. 5, (2003) pp.383-389.

[22] F. Guillemin, and J.V. Leeuwaarden, Rare event asymptotics for a random walk in the quarter plane, Queueing Systems, vol. 67 , no. 1, (2011). pp. 1-32.

[23] J. W. Cohen, On the asymmetric clocked buffered switch, Queueing Systems, vol. 30, no. 3-4, (1998). pp. 385-404.

[24] F. Guillemin, and C. Knessl, and J.V. Leeuwaarden, Wireless Multihop Networks with Stealing: Large Buffer Asymptotics via the Ray Method, Siam Journal on Applied Mathematics, vol. 71, no. 4, (2011). pp. 1220-1240.

[25] I. J-B. F. Adan, V. Houtum, J. Wessels, and Z. Whm, A compensation procedure for multiprogramming queues, Operations-Research-Spektrum, vol. 15, no. 2, (1993). pp. 95-106.

[26] V. G-J. Houtum, Lectures on Riemann surfaces, Technische Universiteit Eindhoven.(1995)

[27] I. J-B. F. Adan and J. Wessels, and W. H. M. Willem, A compensation approach for two-dimensional Markov processes, Advances in Applied Probability, vol. 25, no. 4, (1993). pp. 783-817.

[28] O. Boxma, and G-J. V. Houtum, The compensation approach applied to a $2 \times 2$ switch, Probability in the Engineering and Informational Sciences, vol. 7, no. 4, (1993) pp.471-493.

[29] I. J-B. F. Adan and O. Boxma, and J. Resing, Queueing models with multiple waiting lines, Queueing Systems, vol. 37, no. 1-3, (2001). pp. 65-98.

[30] H. Nassar, and H. Al-mahdi, Queueing analysis of an ATM multimedia multiplexer with non-pre-emptive priority, IEE Proceedings-Communication, vol. 150, no. 3, (2003). pp. 189-196.

[31] O. Forster, and B. Gilligan, Lectures on Riemann surfaces, vol. 81, Springer-Verlag, New York.(1981)

[32] P. Flajolet, and R. Sedgewick, Analytic combinatorics, Cambridge University press.(2009)

[33] EL-S. El-Hady, W. Förg-Rob, H. Nassar, On a Functional Equation Arising from a Network Model, Appl. Math, vol. 11, no. 2, (2017) pp. 363-372.

[34] L. Klimczak, Two constant sign solutions for a nonhomogeneous Neumann boundary value problem, Ann. Univ. Paedagog. Crac. Stud. Math. 14, pp. 47-62, (2015) 
[35] N. I. Muskhelishvili, and J. R. Radok, Singular integral equations: boundary problems of function theory and their application to mathematical physics, Courier Corporation.(2008)

[36] Z. Nehari, Conformal mapping, Courier Corporation.(1975)

[37] H. Kober, Dictionary of conformal representations, Dover New York, vol. 2.(1957)

[38] C. Babbage, Examples of the solutions of functional equations, Paperback reissue of the 1820 original, Cambridge University Press, (2013). 